\title{
Article
}

\section{Exosome TDP-43 profile in canine model of ALS: A preliminary study in developing surrogate biomarker}

\author{
Penelope Pfeiffer ${ }^{1}$, Joan R. Coates ${ }^{2}$, Andrew Kennedy ${ }^{3}$, Kyleigh Getchell ${ }^{3}$, Edina Kosa ${ }^{3}$ and Abdulbaki Agbas ${ }^{3,4 *}$ \\ 1 Mount Sinai Hospital, Chicago IL; pburikas@gmail.com \\ 2 University of Missouri-Columbia, MO; coatesi@missouri.edu \\ 3 Kansas City University, Kansas City MO; ekosa@kansascity.edu; aagbas@kansascity.edu; Andrew.Ken- \\ nedy@kansascity.edu; Kyleigh.getchell@kansascity.edu \\ 4 Heartland Center for Mitochondrial Medicine, Kansas City KS \\ * Correspondence: aagbas@kansascity.edu; Tel.: +1 816-654-7614
}

\begin{abstract}
Blood-based biomarkers are much-needed diagnostic and prognostic tools for ALS. Canine degenerative myelopathy (DM) is recognized animal disease model to study the biology of human ALS. Serum derived exosomes are potential carrier that transport intercellular hormone-like messengers, together with their stability as carrier of proteins and RNA, make them ideal as biomarkers for a variety of diseases and biological processes. We study exosomal TDP-43 pattern as a surrogate biomarker that reflects biochemical changes in central nervous system. We isolated exosomes from canine serum using commercial exosome isolation reagents. TDP-43 and SOD1 profile in spinal cord homogenate lysate and that of serum-derived exosomes were found elevated in dogs with DM. We conclude levels of spinal cord TDP-43 and serum-derived exomes were similar in TDP-43 profiling, which warrant further investigation of disease sensitivity and specificity for establishing as a blood-based biomarker in canine DM.
\end{abstract}

Keywords: neurodegeneration; serum; spinal cord; dog; Degenerative myelopathy; amyotrophic lateral sclerosis

\section{Introduction}

The aberrant protein aggregation in motor neurons is the hallmark of ALS. Misfolded mutant $[\mathrm{Cu} / \mathrm{Zn}]$ superoxide dismutase (SOD1) protein is believed to contribute to the ALS, yet the role of misfolded non-mutant SOD1 in the disease progress is unclear. Recently, chemically modified aberrant Transactive Response DNA Binding Protein 43 (TDP-43) species were found to represent a major accumulating protein in neuronal cytoplasmic inclusions and in exosomes in Frontotemporal Lobar Degeneration (FTLD) and in ALS patients. Exosomes are nano-size membranous vesicles that contains several macromolecules including aberrant pathological proteins. The bio-molecular link and clinical similarities between human ALS and canine DM lead us to propose that human ALS hallmark signature proteins (i.e., SOD1 and TDP-43) have the same or similar profile in canine DM. If so, the same approach may be apply for human ALS that will lead us to establish a reliable blood-based biomarker. ALS is a progressive disease that directly affects motor neurons, leading to loss muscle function. About $5-10 \%$ of the inherited forms of ALS are linked to a mutation in the SOD1 gene (SOD1)

ALS is incurable and fatal and patients survive for 3-5 years once diagnosed [1]. In most patients, the symptoms begin in the lower limbs. Patients often complain of tripping, stumbling, foot drop, or a "slapping" gait. Upper limb involvement includes decreased dexterity in the fingers, cramping, stiffness, weakness and wrist drop. The diagnosis early in the onset of ALS is very difficult, which can delay time to treatment [2]. Establishing a blood-based biomarker(s) will help clinicians with early diagnosis and initiating timely 
treatment. Timely pharmaceutical intervention will improve the quality of life and patient lifespan.

Animal models of ALS have used rodents with mutated SOD1 to study the pathology of the disease [3]. There are limits in use of the rodent model as disease progression is very short (3-6 months) and is based on overexpression of the human gene for mutant SOD1. In addition, peripheral biomarker studies are difficult to perform in a longitudinal course due to limited access to serial samples of serum/exosomes in mice due to small volume of blood. Canine degenerative myelopathy (DM) is a late adult neurodegenerative disease accompanied by (SOD1) mutations (SOD1:c.118A,SOD1:c52T) and protein aggregation [4]. Early clinical signs are general proprioceptive ataxia and spastic upper motor neuron paresis in the pelvic limbs with progression to flaccid tetraplegia and dysphagia [5-7]. DM and ALS are therefore characterized by progressive multisystem neurodegeneration involving upper and lower motor neurons. Histologic similarities include astrogliosis in the spinal cord, degeneration and loss of myelinated axons of the spinal cord and peripheral nerves and neurogenic muscle atrophy $[5,6]$. The proposed study will discuss the exploration of exosomal TDP-43 as apotential translational biomarker in DM and ALS. It is practical to complete serial blood sampling of serum/exosome in dogs for longitudinal study as potential biomarker.

An abnormal accumulation of TDP-43 in the cytoplasm and platelets in ALS [8] and the protein is misfolded or post-translationally modified (i.e., hyper-phosphorylation and ubiquitination) $[9,10]$. It is thought that the accumulation of TDP-43 in the cytosol causes the loss of function in affected neurons [11]. However, it is not known the amount of peripheral TDP-43 originates from the central nervous system (CNS); therefore, introducing the exosomal TDP-43 evaluation in a fashion that encapsulated TDP-43 in exosome better reflects the aberrant TDP-43 rather than general population of TDP-43.

\section{Results}

2.1. Dogs with DM spinal cord homogenate show elevated TDP-43 and SOD1 in thoracic region

Thoracic region of spinal cord from companion dogs with DM $(n=4)$ and from age matched non-DM affected control dogs $(n=4)$ were obtained from a tissue archive at the University of Missouri College of Veterinary Medicine. The signalment of dogs used in this study listed in Table-1 in Supplemental Data. The $-80^{\circ} \mathrm{C}$ frozen spinal cord tissue was homogenized and stored at $-80^{\circ} \mathrm{C}$, and later then subjected to $10 \%$ SDS/PAGE followed by western blot analysis. Proteins were immunoprobed with anti-TDP-43 Ab (1/1000 dilution). The intensity of the bands were analyzed by an image analyzer. Figure-1 showes that TDP-43 protein levels are elevated in DM spinal cord region as compare to that of control dogs. We applied one simple $t$ test analysis and found differences between two groups were significant $(\mathrm{P}<0.01$ ). This observation is in aggrement of other published data that TDP-43 accumulation was observed in human spinal cord [9, 12] SOD1-immunoreactive aggregates were observed in ventral horn motor neurons [13]. In this study we confirmed spinal cord SOD1 aggregation profile occured in canine with DM (Fig.2). We considered SOD1 aggregation profile as disease control. 


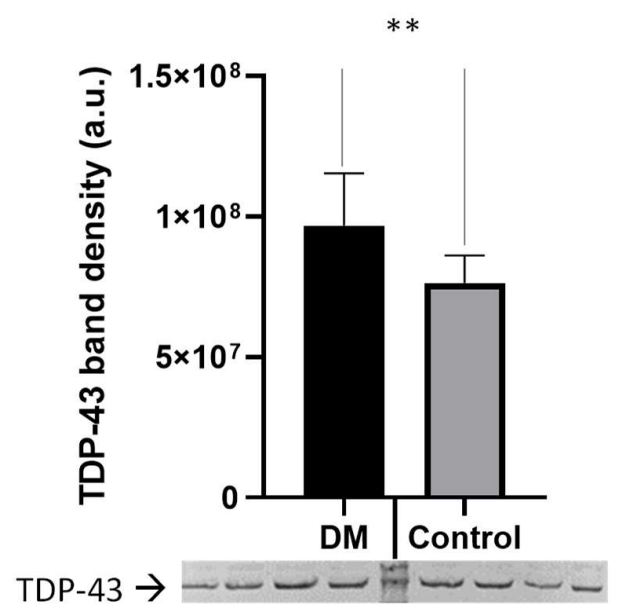

Figure 1. Total TDP-43 levels of thoracic region of spinal cord homogenate. DM affected dogs ( $n=4$, Supplemental Data / Table-1: dog \# 1,2,3,4) showed elevated levels of TDP-43 compared to control $\operatorname{dogs}(\mathrm{n}=4$, Supplemental Data / Table- 1 : dog \#5,6,7,8) [One- sample t-test $(\mathrm{P}<0.01)$ ]. The protein band intensities were normalized to total protein staining.

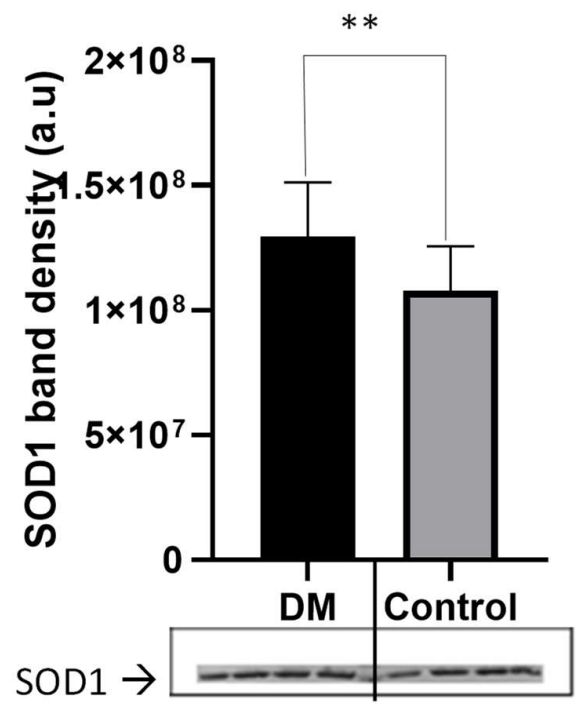

Figure 2. SOD1 levels of thoracic region of spinal cord homogenate. DM affected dogs ( $\mathrm{n}=4$, Supplemental Data / Table-1: dog \# 1,2,3,4) showed elevated levels of SOD1 as compare to control dogs $(\mathrm{n}=4$, Supplemental Data / Table-1: dog \# 5,6,7,8) [one-sample t-test $(\mathrm{P}<0.01)$ ]. The protein band intensities were normalized to total protein staining.

\subsection{Exosomal TDP-43, phosphorylated TDP-43, and SOD1 are elevated in canine with DM}

Frozen serum samples from dogs were obtained from a tissue archive at the University of Missouri College of Veterinary Medicine. The signalment of dogs used in this study listed in Table-2,3 in Supplemental Data. Exosomes were isolated from serum by means of vesicle precipitation methods described in miRCURY exosome isolation kit (Qiagen \#76603). Isolated exosome lysates were subjected to SDS/PAGE (4-20\% gradient gel) followed by immuno blot analysis. The protein bands were probed with both anti-TDP-43 and anti-pTDP-43 Ab (1/100 dilution). The intensity of the protein bands were analyzed by an image analyzer (LI-COR Odyssey iInfrared analyzer). Figure-3A and 3B showed 
that both total TDP-43 and its phosphorylated derivative (pTDP-43) are elevated in exosomes of dogs affected with DM as compared to that of control dogs. Difference between $\mathrm{DM}$ affected and control dogs was found significant $(\mathrm{P} \leq 0.001)$. These results suggest that exosomes can be used to assess biomarker proteins such as TDP-43 and its phosphorylated derivatives.

A

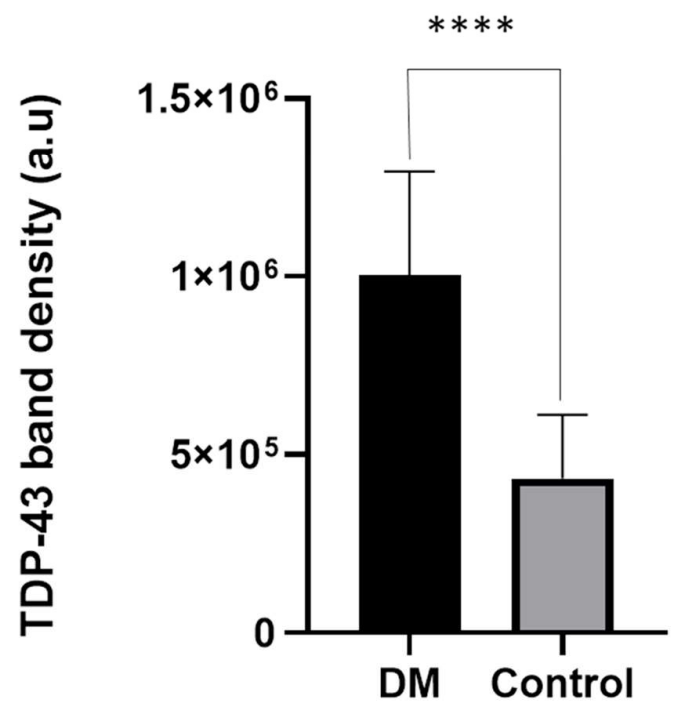

B

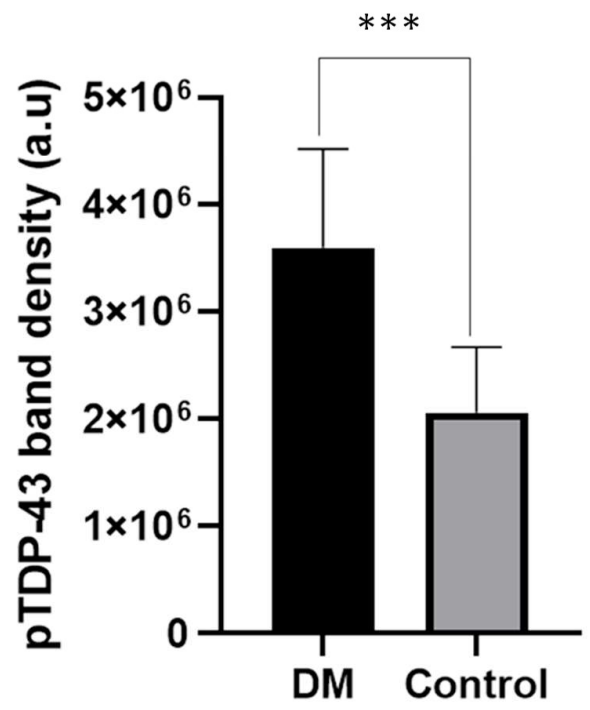

Figure 3. Serum derived exosomal total TDP-43 and phosphorylated TDP-43 profile in SM affected dogs. Both total TDP43 (A) and phosphorylated TDP-43 (pTDP-43) (B) levels were elevated in serum-derived exosomes from DM affected dogs ( $\mathrm{n}=12$; Table-2 Supplemental Data: dog \# 1,2,3,4,5,6,7,8,9,10,11,12) compared to control dogs ( $\mathrm{n}=10$; Table-3 Supplemental Data: $\operatorname{dog} \# 1,2,3,4,5,6,7,8,9,10)$. One sample $t$-test analysis $\mathrm{P}<0.0001$ for total TDP-43 and $\mathrm{P}<0.001$ for pTDP-43. Original immunoblot analysis pertinent to this figure provided in Supplemental Fig.1 and Fig.2. The protein band intensities were normalized to total protein staining.

SOD1 proteins in canine serum- derived exosomes exhibit a heterogenic profile (i.e., momomer, trimer and tetramer) (Fig.4). We have considered soluble monomers ( $25 \mathrm{kDa})$ in our data analyses and graphing. Dogs affected with DM serum exomes had elevated SOD1 proteins when compared to control dogs. The difference between two groups were significant based on unpaired t-test analysis $(\mathrm{P}<0.05)$.
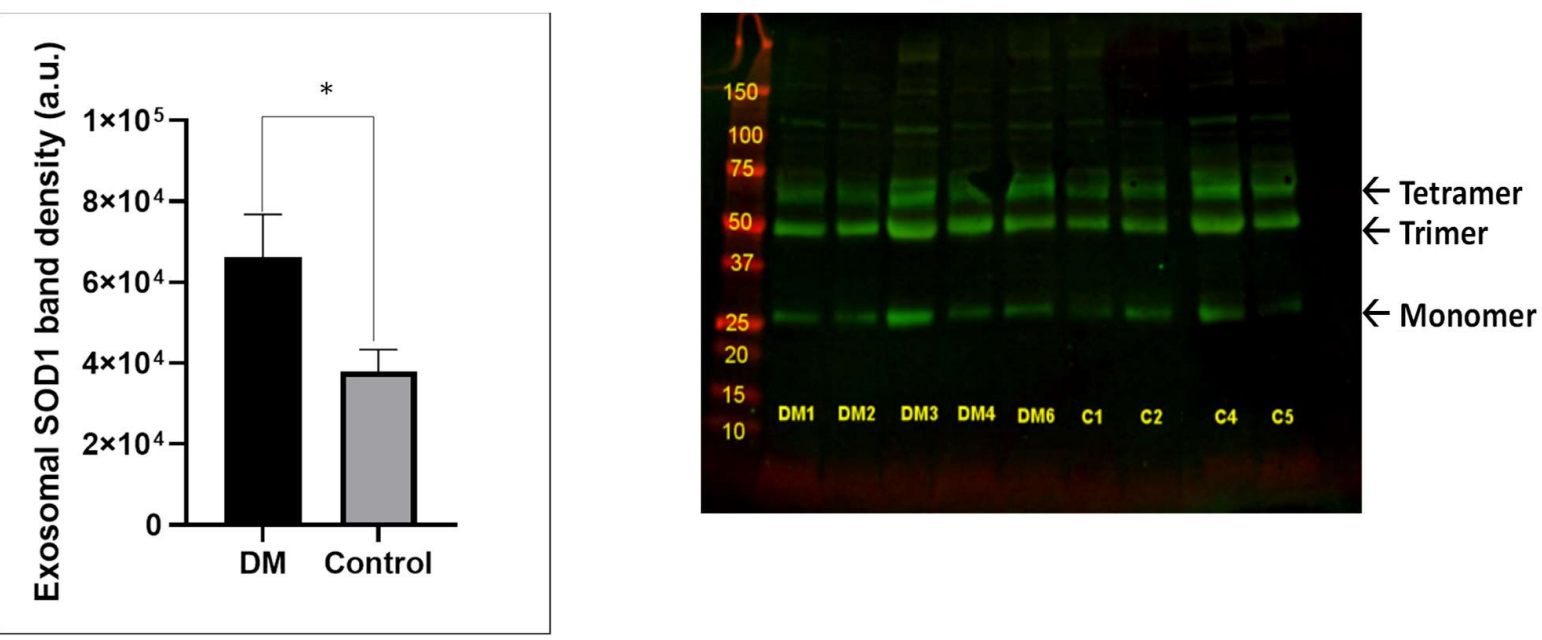
Figure 4. Serum derived exosomal SOD1 profile in dogs with DM. (A) Exosomal SOD1 levels appear to be elevated in dogs with DM. The difference between control $(n=4$, Table-3 Supplemental Data ; dog \#1,2,4,5) and DM ( $n=5$; Table-2 Supplemental Data: $\operatorname{dog} \# 1,2,3,4,6)$ groups did achieve statistically significance ( $p<0.05$ ). (B) Monomer SOD1 proteins bands were analyzed and graphed. The protein band intensities were normalized to total protein staining.

\section{Discussion}

Complexity of biosample matrix and invasive nature of tissue sampling led investigators to develop peripheral tissue-based biomarkers. Blood-based biomarker initiatives gain most popularity due to easy access for sampling with a minimal invasion (i.e., venous puncture), access to several blood cells and subcellular structures. Once developed and validated, blood-based biomarkers will be utilized for diagnosis, monitoring the prognosis, and monitoring the treatment of ALS. In this study, we demonstrated the use of exosomes and its ALS hallmark proteins (i.e., TDP-43, pTDP-43 and SOD1) and compared to thoracic spinal cord homogenate. We chose the thoracic region because the disease pathology of canine DM is most severe in this region [14]. This region also reflects more TDP43 accumulation relevant to pathology of ALS [15].

The dog cohort was consisting of animals with degenerative myelopathy (DM) and age matched non-DM affected dogs for serum exosome isolation. These analyses strengthen the positive correlation between spinal cord- and blood-derived exosomal TDP-43 and SOD1 profile and eventually assign exosomes as validated surrogate biomarker. This will translate to adopt human blood-derived exosome to establish a valid biomarker for human ALS.

In this study, we have demonstrated the method how to isolate exosomes from serum, analyze exosomal TDP-43 and pTDP-43 protein profile and compare to that of spinal cord tissue (thoracic region) homogenate from the different cohort of canine with DM as part of proof of concept studies. TDP-43 and SOD1 are known as hallmark proteins for ALS $[9,10,16]$. We demonstrated total homogenates of spinal cord TDP-43 levels were elevated in canine with DM (Fig.1). We also showed SOD1 profile as positive control indicating that both proteins were elevated (Fig.2). Our next step will be to demonstrate sensitivity and specificity to establish biomarker reliability.

Exosomes are small stable single-membrane organelles and carry selected proteins, lipids, nucleic acids, and glycoconjugates [17]. Exosome biogenesis is a mechanism of protein quality control [17]. They are emerging as key mediators of communication and waste management among neurons, glial cells and connective tissues during normal and diseases conditions [18]. Although exosomes are being developed as therapeutic agents in multiple disease models, they can also serve as surveillance agents by transporting aberrant proteins like hyper-phosphorylated TDP-43 or cytosolic TDP-43 that could not reenter into nucleus. Canine DM allowed us to establish the link between spinal cord and serum/plasma derived exosomes in terms of TDP-43 biochemistry. We have observed increased TDP-43 and SOD1 levels in exosomes derived from dog affected with DM. We have used SOD1analysis to further support spinal cord and serum exosome cross-talk in terms of the content TDP-43 and its derivative. SOD1 protein profile was interesting. We observed at least three SOD1 species (monomer, trimer, and tetramer) although SOD1 resolved under the reducing conditions. In our previous study because of their small size, exosomes can easily pass blood brain barrier and serve as accessible biomarkers of neuronal dysfunction [19]. Thus, we may conclude that spinal cord TDP-43 may be transported by exosomes and TDP-43 loaded exosomes appeared in blood [20]. We are aware of that exosomes derived from serum represents a global exosomes including neuronal derived exosomes (NDE). Currently, we are developing an approach to specifically isolate NDE population from global serum and spinal cord tissue derived exosomes and analyze their content in terms of TDP-43 and its derivatives.

\section{Materials and Methods}




\subsection{Animals}

Previously $-80^{\circ} \mathrm{C}$ frozen spinal cord tissues from thoracic region and serum samples were obtained from Dr. Joan R. Coates at the College of Veterinary Medicine, University of Missouri-Columbia. Tissues were harvested from various breeds with diagnosed DM and age-matched dogs not affected with DM dogs (Table 1 Supplemental Data). ALL DM affected dogs were homozygous for the SOD1 E40K mutation [7].

\subsection{Isolation of exosomes from canine serum}

The isolation of exosomes from serum of DM and control dogs was performed using a commercially available particle precipitation method following manufacturer's protocol (miRCURY Exosome Serum/Plasma Kit, Qiagen \#76603). Exosome isolation procedure is based on the capture of water molecules, which otherwise form the hydrate envelope of particles in suspension. Mixing the starting sample with the a proprietary precipitation buffer diminishes the hydration of the subcellular particles and allows precipitation of even particles smaller than $100 \mathrm{~nm}$ with a low-speed centrifugation step [21] To remove residual cells, debris, platelets, large micro vesicles etc., the serum samples were first pelleted and the supernatant filtered. The samples were incubated with a proprietary precipitation buffer for 1 hour at $4^{\circ} \mathrm{C}$. After a 30 minutes low speed centrifugation the exosome pellets were re-suspended for further characterization. Exosomes yield was verified by immunoprobing assay using an antibody recognizes exosome surface protein, TSG101 (tumor susceptibility 101 protein). Exosome suspension was aliquoted and kept in $-20^{\circ} \mathrm{C}$ for storage.

\subsection{Spinal cord homogenate preparation}

50-60 mg thoracic spinal cord tissue (T3 section) were homogenized in an ice-cold buffer $\left(0.32 \mathrm{M}\right.$ sucrose, $0.5 \mathrm{mM} \mathrm{MgSO}_{4}, 10 \mathrm{mM} \varepsilon$-amino-n-caproic acid, protease inhibitor cocktail .1\% v/v, $10 \mathrm{mM}$ HEPES, pH 7.4) in a glass pestle-glass homogenizer (8-10 strokes). The tissue: homogenization buffer ratio was 1 (mg tissue): 15 ( $\mu \mathrm{L}$ buffer). The homogenate was resuspended 8-10 times using a $1 \mathrm{~mL}$ syringe with a $26^{6 / 8}$ gauge needle to shear DNA and liberate nuclear proteins. The homogenate was incubated on ice for 20 minutes followed by centrifuged at $16,000 \mathrm{X} \mathrm{g}$ for 20 minutes at $4^{\circ} \mathrm{C}$. The supernatant was collected into a clean tube. It contains all soluble proteins, including nuclear proteins. 5-10 $\mu \mathrm{L}$ aliquot was saved for protein assay. Sample was aliquoted into $100 \mu \mathrm{L}$ volumes into microfuge tubes and store at $-80^{\circ} \mathrm{C}$ until use.

\subsection{Immunoblotting analysis}

$10 \mu \mathrm{g}$ of exosomal proteins were loaded onto a $4-20 \%$ gradient gel. Electrophoresis run at $100 \mathrm{~V}$ for $75-80 \mathrm{~min}$ until dye-front migrated to $0.5 \mathrm{~cm}$ from the bottom of the gel. Resolved proteins on the gel were transferred onto a PVDF membrane by electro transfer unit at $60 \mathrm{~V}$ for $90 \mathrm{~min}$. The membrane was stained/destained for total protein staining according to manufacturer's protocol (REVERT Total Protein Stain kit, LI-COR, Cat\# 92611016). The membrane was blocked with a blocking agent (SEA BLOCK Blocking, Thermo Scientific, Cat \# UH2788881) for 1hr. at room temperature (RT) followed by overnight incubation with anti- TDP-43 Ab (1:1000 dilution) and anti-SOD1 Ab (1:750 dilution) on an orbital shaker at $4^{\circ} \mathrm{C}$. Next day, the membrane was incubated with Infrared (IR)-tagged antibody for 1-2 hrs. at RT. The protein bands were visualized in an image analyzer (LICOR Odyssey Infrared Imager (Model No. 9120). The intensity of protein bands were normalized based on total protein staining signals and analyzed by Image Studio image analyzing program (V.3.1, Li-COR Biosciences).

Author Contributions: Conceptualization, AA and JRC; methodology, AA, PP, AK,KG, and EK.; formal analysis, $\mathrm{PP}, \mathrm{AK}, \mathrm{KG}, \mathrm{EK}$, and AA; writing - original draft preparation, AA; writing - review and editing, AA and JRC; supervision, AA and EK; All authors have read and agreed to the pub- 
lished version of the manuscript." Funding: This research was funded by Summer Research Fellowship Program (PP) provided by Kansas City University; Intramural grant to support (AA) for student researchers ( $\mathrm{AK}$ and $\mathrm{KG}$ ), and a grant from Kansas City Consortium of Musculoskeletal Disorders (AA and JRC).

Institutional Review Board Statement: The study was conducted according to the guidelines of the Declaration of Helsinki, and approved by the Institutional Review Board (or Ethics Committee) of University of Missouri-Columbia (protocol code 8339 and date of 01/15/2015)

Acknowledgments: Authors acknowledge Kansas City University for supporting student research and providing logistic supports, Robert Liu, DO for providing technical help, and Jessica Sage for training summer research fellows.

Conflicts of Interest: The authors declare no conflict of interest.

\section{REFERENCES}

1. What is ALS? 2014 02/16/2014; Available from: www.alsa.org.

2. Armon, C. Amyotrophic lateral sclerosis. 2014 [cited 2014; Available from: $<$ http://emedicine.medscape.com/article/1170097overview>.

3. Wegorzewska, I. and R.H. Baloh, TDP-43-based animal models of neurodegeneration: new insights into ALS pathology and pathophysiology. Neurodegener Dis, 2011. 8(4): p. 262-74.

4. Awano, T., et al., Genome-wide association analysis reveals a SOD1 mutation in canine degenerative myelopathy that resembles amyotrophic lateral sclerosis. Proc Natl Acad Sci U S A, 2009. 106(8): p. 2794-9.

5. Morgan, B.R., et al., Characterization of intercostal muscle pathology in canine degenerative myelopathy: a disease model for amyotrophic lateral sclerosis. J Neurosci Res, 2013. 91(12): p. 1639-50.

6. Morgan, B.R., et al., Characterization of thoracic motor and sensory neurons and spinal nerve roots in canine degenerative myelopathy, a potential disease model of amyotrophic lateral sclerosis. J Neurosci Res, 2014. 92(4): p. 531-41.

7. Coates, J.R. and F.A. Wininger, Canine degenerative myelopathy. Vet Clin North Am Small Anim Pract, 2010. 40(5): p. 929-50.

8. Sage, J., et al., Use of Capillary Electrophoresis Immunoassay to Serach for Potential Biomarkers of Amyotrophic Lateral Sclerosis in Human Platelets. J.Vis.Exp., 2020. 156(e60638): p. 1-9.

9. Neumann, M., et al., Phosphorylation of S409/410 of TDP-43 is a consistent feature in all sporadic and familial forms of TDP-43 proteinopathies. Acta Neuropathol, 2009. 117(2): p. 137-49.

10. Neumann, M., et al., Ubiquitinated TDP-43 in frontotemporal lobar degeneration and amyotrophic lateral sclerosis. Science, 2006. 314(5796): p. 130-3.

11. Baloh, R.H., TDP-43: the relationship between protein aggregation and neurodegeneration in amyotrophic lateral sclerosis and frontotemporal lobar degeneration. FEBS J, 2011. 278(19): p. 3539-49.

12. Feneberg, E., et al., Detection and quantification of novel C-terminal TDP-43 fragments in ALS-TDP. Brain Pathol, $2020:$ p. e12923.

13. Katz, M.L., et al., Cervical spinal cord and motor unit pathology in a canine model of SOD1-associated amyotrophic lateral sclerosis. J Neurol Sci, 2017. 378: p. 193-203.

14. Averill, D.R., Jr., Degenerative myelopathy in the aging German Shepherd dog: clinical and pathologic findings. J Am Vet Med Assoc, 1973. 162(12): p. 1045-51.

15. Noto, Y., et al., Elevated CSF TDP-43 levels in amyotrophic lateral sclerosis: specificity, sensitivity, and a possible prognostic value. Amyotroph Lateral Scler, 2011. 12(2): p. 140-3.

16. Neumann, M., et al., TDP-43-positive white matter pathology in frontotemporal lobar degeneration with ubiquitin-positive inclusions. J Neuropathol Exp Neurol, 2007. 66(3): p. 177-83.

17. Pegtel, D.M. and S.J. Gould, Exosomes. Annu Rev Biochem, 2019. 88: p. 487-514.

18. Song, Z., et al., Brain Derived Exosomes Are a Double-Edged Sword in Alzheimer's Disease. Front Mol Neurosci, 2020.13 : p. 79.

19. Saeedi, S., et al., The emerging role of exosomes in mental disorders. Transl Psychiatry, 2019. 9(1): p. 122.

20. Sproviero, D., et al., Pathological Proteins Are Transported by Extracellular Vesicles of Sporadic Amyotrophic Lateral Sclerosis Patients. Front Neurosci, 2018. 12: p. 487.

21. miRCURY Exosome kits handbook, Qiagen, Editor. 2017. p. 7-8. 